\title{
PLATING VERSUS NAILING IN ASEPTIC NONUNION SHAFT TIBIA MANAGEMENT
}

\author{
Mostafa Meshneb $^{(*)}$, Ahmed Saleem, Hassan Noaman Abd-El-Rahman Hafez \\ Orthopaedic dept, Faculty of Medicine, Sohag University, Sohag, Egypt \\ "E-mail: mostafameshneb2@gmail.com
}

Received 1/3/2021

Accepted 28/5/2021

\begin{abstract}
The purpose of this study is to compare results of usage of reamed interlocking nail versus plating in management of aseptic non- union fracture shaft tibia. 20 patients with aseptic non union tibia were managed in our department between June 2015 and June 2016. Patients were divided randomly in this retrospective study into two groups: plating group (10 patients) and nailing group (10 patients). Mean age of patients (plating group) was 40.3 years old, while in (nailing group) was 30.3 years old. Iliac bone graft was used in 4 patients (plating group) and 3 patients (nailing group). Initial fracture was simple in 8 cases (4 in plating group and 4 in nailing group) and compound in 12 cases (6 in plating group and 6 in nailing groups). All patients were followed up in our patient clinic; with a mean period of follow up was 36.9 weeks. 19 cases healed and one case lost from (plating group). Mean period of healing was 6.7 months (plating group) while 4.7 months in (nailing group). According to Olerud and Molander score, 2 patients excellent, 2 good and 5 fair (plating group), while 1 patients excellent, 7 good and 2 fair (nailing group). No significant complications rate difference was found between the two groups. Reamed interlocking nail can obtain higher union rate, shorter time to union incidence and less complications rate than plating in the treatment of aseptic non union fracture shaft tibia.
\end{abstract}

Keyword: Aseptic, Tibia non- union, Plating, Nailing, Grafting.

\section{Introduction}

Non-union of tibial fractures is a relatively common problem and its incidence is higher than in other long bone fractures [1]. The incidence of tibialnon union is $2-10 \%$ of all tibia fracture; incidence is higher in high energy injuries and open fractures. The Weber-Cech [2] classification is the one most widely used in this system. Most patients with this problem have been incapacitated for between six to twelve months
[3]. Furthermore scarring from previous surgery and generalized osteopenia secondary to prolonged immobilization are important factors to be considered in further surgical management. Reamed intramedullary nailing offers the advantages of stable fixation, realignment of the intramedullary canal, and dynamic compression at the nonunion site by early weight bearing. Success rates as high as $94-100 \%$ have been 
reported in some series [4,5]. However, generalized intramedullary osteomyelitis following reaming is the most worrying complication. Plating has the advantage of stable fixation in non union tibia where the fracture is distal or proximal. Soft tissue complications and metal failure are most common complications of plating. We conducted this study to compare the outcome of the reamed intramedullary procedure and plating in aseptic nonunion tibia

\section{Patients and Methods}

\subsection{Patients data}

Between June 2015 and June 2016, twenty consecutive patients with aseptic nonunion of the fracture shaft tibia were treated in our orthopedic department in Sohag medical school after taking ethical committee acceptance in our school. Patients were divided by blind randomization in two groups: Ten patients were managed by plating; other ten patients were managed by reamed interlocking nail. Their average age was 33.7 years (range 17 to 65 years). There were thirteen males and seven females patients. The average follow-up was nine months (range between 7 to 18 months). The initial fracture was closed in eight patients (4 patients in each group) and open in twelve patients (6 patients in each group). Moreover, five fractures were in the lower third area of the meta-diaphysealregion, the other ten were in the mid-shaft and five fractures in proximal third fractures, tab. (1). The primary treatment had been with external fixator in four cases, plating in two, plaster cast in three patients and interlocking nail in one patient. The patients had to undergo an average of two surgical procedures prior to non-union treatment. Seven patients underwent illac bone grafting for bone loss. There were thirteen atrophic, five hypertrophic nonunions and two pseudoarthrosis. The average interval between initial fracture and treatment of non-union was six months (range 3-14) months.

Table (1) comparosn between No of pationt according to level of fructure

\begin{tabular}{|l|c|c|}
\hline Fracture level & $\begin{array}{c}\text { Number of patients } \\
\text { with plate }\end{array}$ & $\begin{array}{c}\text { Number of } \\
\text { patients with nail }\end{array}$ \\
\hline Proximal shaft & 4 & 1 \\
\hline Mid shaft & 3 & 7 \\
\hline Distal shaft & 3 & 2 \\
\hline Total & 10 & 10 \\
\hline
\end{tabular}

\subsection{Surgical procedure}

The erythrocyte sedimentation rate and the C-reactive protein level were within normal limits in all patients prior to surgical intervention. Reamed intramedullary technique was attempted in ten cases. The proximal entry of the guide wire was performed through a patella tendon splitting incision. A non-ball tip guide wire was introduced until the nonunion area, and the fracture alignment was then confirmed by image intensifier. The reamer was carefully introduced to beyond the fibrous union, ensuring the disruption of the fibrous tissue to facilitate the further advancement of the guide wire. This procedure was a crucial part of the surgery to ensure the guide wire could be passed across non-union sites. Reaming of the intramedullary canal was performed in 0.5 $\mathrm{mm}$ increments to the largest diameter. The nails were locked distally and proximally with two screws for stability. On the other hand, plating technique was done in other ten patients. Meticulous dissection was done at fracture site to protect soft tissue, good refreshing of bony edges was done and good alignment of bone with stable fixation was done by narrow locked plate in 3 patients and anatomical locked plate in 7 patients.

\subsection{Postoperative care}

Weight bearing in plating delayed up to 
six weeks. Touch weight bearing was allowed in $2^{\text {nd }}$ postoperative day in nailing group then patients were encouraged to partial weight bear using crutches All patients in two groups were followed up by clinical examination included physical examination, neurovascular examination, assessment of axial alignment, and a functional analysis. Rotational alignment was assessed clinically by checking the relationship of the intercondylar axis with the intermalleolar axis and comparing with that of the normal side. Functional recovery was assessed using Olerud-Molanderankel score [6]. Olerud-Molanderankel score of 91 to 100 points was considered excellent; 61-90 points, good; 31-60 points, fair; and less than 31, poor. At every visit patients were examined using $\mathrm{x}$-ray to asses fracture union and clinically regarding knee and ankle function, walking ability, deformity and limb length discrepancy.

\section{Results}

Union was defined as radiological evidence of solid bridging callus formation and painless limb on full weight bearing. All non-union of tibia fractures healed at average of 6 months (range 3-12 months) except one patient lost. Table (2) showing difference in healing incidence in the two groups. Average union time in nailing group was 4.7 months and 6.7 months in plating group, fig $(1,2)$. Eight cases with no complication (nailing group), One patient (nail group) presented by mal rotation deformity (15 degree of external rotation of leg) and the other case presented by superficial infection at site of distal locking screws and was managed by serial debridement and locking screws removal. On the other hand, six cases with no complications (plating group): two cases (plating group) presented by superficial infection (one was at site of plate, other at site of iliac graft). Both of them were managed by serial debridement and parenteral antibiotics according to culture and sensitivity. Plate exposure happened in one case. This case managed by local rotational flap. Three cases were excellent, nine cases were good and seven cases were fair according to Olerud and molander score (excellent more than 91, good between 61 and 90, fair between 31 and 60 and poor less than 31 ), tab. (3).

Table (2) showing difference in healing incidence in the two groups

\begin{tabular}{|l|c|c|}
\hline \multicolumn{1}{|c|}{$\begin{array}{c}\text { Union } \\
\text { incidence }\end{array}$} & $\begin{array}{c}\text { Number of patients } \\
\text { with plate }\end{array}$ & $\begin{array}{c}\text { Number of } \\
\text { patients with nail }\end{array}$ \\
\hline$<6$ months & 4 & 6 \\
\hline 6-9 months & 3 & 4 \\
\hline$>9$ months & 2 & 0 \\
\hline Total & 9 & 10 \\
\hline
\end{tabular}

Figure (1) shows female patient 55 years old presented by aseptic non union tibia managed by plating and grafting, a. preoperative $\mathrm{x}$ ray, $\underline{\mathbf{b}}$. postoperative $x$ ray, $\underline{\text { c. five }}$ months follow up
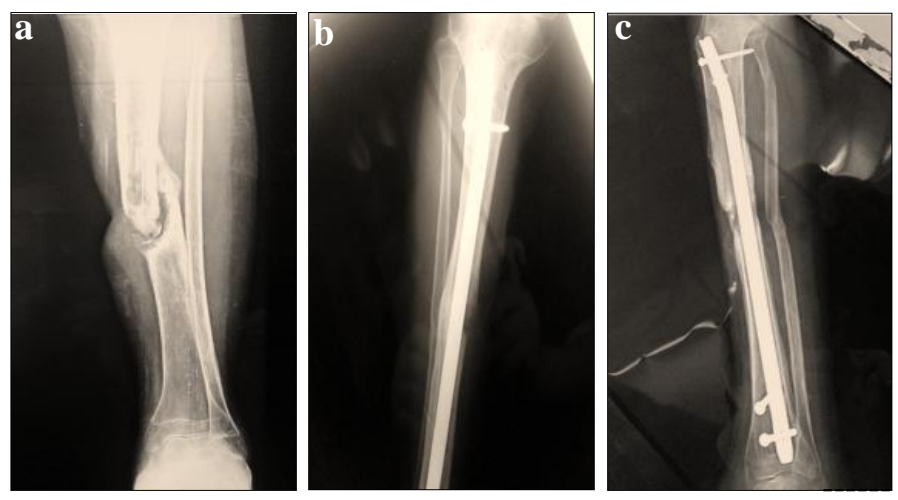
Figure (2) shows male patient 18 years old presented by aseptic non union tibia managed by interlocking nail and grafting, a. preoperative $\mathrm{x}$ ray, $\underline{\mathbf{b}}$. six weeks follow up, c. five months follow up
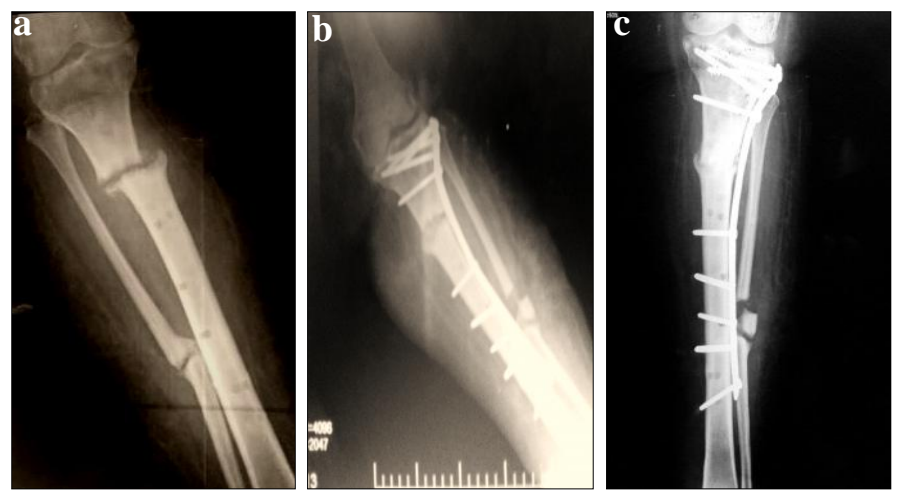

Table (3) showing difference in two groups

\begin{tabular}{|l|c|c|}
\hline $\begin{array}{c}\text { Olerud and } \\
\text { Molander score }\end{array}$ & $\begin{array}{c}\text { Number of patients } \\
\text { with plate }\end{array}$ & $\begin{array}{c}\text { Number of } \\
\text { patients with nail }\end{array}$ \\
\hline Excellent & 2 & 1 \\
\hline Good & 2 & 7 \\
\hline Fair & 5 & 2 \\
\hline Poor & 0 & 0 \\
\hline Total & 9 & 10 \\
\hline
\end{tabular}

\section{Discussion}

The main causes of long bone fracture eventuating in nonunion are instability of the fracture ends, insufficient bony contact and loss of blood supply [7]. Several surgical treatments have been advocated for the treatment of non union fracture tibia including compression plating, fibular osteotomy, cancellous bone graft, Illazrof method and interlocking nail. The main cause of non union in this study is loss of blood supply as fourteen cases are atrophic non union. Ten patients managed by plates, other ten patients managed by interlocking nail. Seven patients were managed by autogenus illac grafting. Ave-rage follow up in our study was 9 months (range 7 to 18 months). Twenty patients achieved bone healing. Mean time of incidence of union was 6 months (range between 3 to 12 months). Ten patients achieved union in less than 6 months, seven patients between 6-9 months and three patients in more than 9 months. While in $\mathrm{CC}$ Wu et al [8], all 31 patients were treated by rea- med interlocking nailing. 28 patients were followed up for at least one year (range 1.0-5.2 years; median, 2.2 years), while 3 were lost contact. All 28 tibialnonunions healed uneventfu-lly, giving a $100 \%$ union rate. The median union period was 4.5 months (range, 3.0-7.5 months). No extra cancellous bone graft was supplemented. While in WI Faisham, et al [9], all twelve patients with aseptic non-union of the tibia were treated with the reamed interlocking technique. The average follow-up was eight months (range between 6 to 36 months). All non-union of tibial fractures healed at average of 16 weeks (range 1230 weeks). Two patients underwent bone grafting for bone loss but failed to unite. In Galal Z. Said, et al [10]. Thirty patients compl-aining of non union tibia were managed by plating (tibia profibula technique). Twenty two patients showed union at three months' follow-up. Of the remaining patients, four united in four months, and two united in six months. Two patients showing no radiological signs of 
healing at four months' follow-up were bone grafted, and united two months later. The mean healing time was 3.5 months. The duration of follow-up ranged between ten and 38 months (average 26 months). Grafting were done in 26 cases. In our study, average union time is 4.7 months (nailing group), one case (nailing group) is complicated by malro-tation (15 degree external rotation), other case in this group complicated by infection at distal locking screw. So incidence of complications in this group is $20 \%$. While average time of union was 6.7 months range between (3-12 months) (plating group). Two cases complicated by deep infection, serial debridement was done with culture and sensitivity test. Other case complicated by plate exposure, a local flap was done. Other one case complicated by infection at grafting site. So 4 cases $(40 \%)$ were complicated. So reamed interlocking nail give us best results and less complication rates. Interlocking intramedullary nailing with reaming offers the advantages of stable fixation, adequate alignment, minimum shortening, early weight bearing and high rate of union. Intramedullary nailing with reaming however is not technically simple. The procedure requires the use of specialized instruments and techniques, including fluoroscopy image assistance. The risk with intramedullary nailing is the threat of infection, which is higher in cases with open fracture treated previously with external fixator and previous infection. Thorough evaluation before surgery and longer antibiotic cover in these cases will reduce the infection risk. While in Shih CH, et al [11]. Among 105 tibia shaft aseptic nonunion followed for a mean of 32 months, 30 were treated with dynamic compression plating and 75 were treated with either Küntscher or Grosse-Kempf interlocking intramedullary nailing. With the plating technique, the union rate was $90.0 \%$ (27/30), the period of time to union was $5.2^{+} / 1.7$ months, the complication rate was $16.7 \%(5 / 30)$, the infection rate was $13.3 \%$ (4/30), and the surgical time was $110 \% 30$ minutes. With the intramedullary nailing technique, the union rate was $93.3 \%(70 / 75)$, the period of time to union was $5.0^{+} \% 1.6$ months, the complication rate was $17.3 \%(13 / 75)$, the infection rate was $13.3 \%(10 / 75)$, and the surgical time was $70 \% 30$ minutes. While in Fariborz, et al 75 adult ( $>18$ years) patients suffering from long bone (Femur, Tibia, Humerus and Ulna) non-union fracture who were randomly assigned to receive $5 \mathrm{~mL}$ PRP $(\mathrm{n}=37)$ or $5 \mathrm{~mL}$ normal saline as placebo $(n=38)$ in the site of fracture after intramedullary nailing or open reduction and internal fixation (ORIF) along with autologous bone graft .The healing rate was significantly higher in PRP group compared to placebo $(81.1 \%$ vs. 55.3\%; $\mathrm{p}=0.025)$. The limb shortening was significantly higher in those who received placebo $(2.61 \pm 1.5$ vs. $1.88 \pm 1.2$ $\mathrm{mm} ; \mathrm{p}=0.030$.) However, the weakness in our study is low number of patients so we recommend larger number of patients for future studies.

\section{Conclusion}

Reamed interlocking nail can be used in the management of aseptic on union tibia and give better results than plating technique. However nailing is technically requiring the availability of appropriate tools and surgical implants, Careful follow up of the patients is recommended. This technique confirmed by our results minimizes the complication rate and facilitates early return to normal activities of the patient. 


\section{References}

[1] Boyd, H., Lipinsky, S., Willey, J. (1961). Observation on nonunion of the shafts of long bones with statistical analysis of 842 patients. J. Bone J. Surg. 43 (2): 159-168.

[2] Weber, B. \& Čech, O. (1976). Pseudarthrosis: Pathophysiology, biomechanics, therapy, results, Grune \& Stratton, Switzerland

[3] Sledge, S., Johnson, K., Henley, M., et al. (1989). Intramedullary nailing with reaming to treat non-union of the tibia, J. Bone Joint Surg Am. 71 (7): 1004-1019.

[4] Clancey, G., Winquist, R., Hansen, S. (1982). Nonunion of the tibia treated with Kunt, cher intramedullary nailing. Clin Orthop Relat Res. 167: 191-196

[5] Megas, P., Panagiotopoulos, E., Skriviliotakis, S., et al. (2001). Intramedullary nailing in the treatment of aseptic tibial nonunion. Injury. 32: 233-239.

[6] Olerud, C., Molander, H. (1984). A scoring system for symptom evaluation after ankle fracture. Arch Orthop Trauma Surg. 103: 190-194.

[7] Weresh, M., Hakanson, R., Stover, M., et al. (2000). Failure of exchange rea- med intramedullary nails for un united femoral shaft fractures. J. Orthop Trauma. 14: 335-338.

[8] Wu, C. (2003). Reaming bone grafting to treat tibial shaft aseptic nonunion after plating. J. Orthop Res. 11 (1): 16-21.

[9] Faisham, W., Sulaiman, A., Sallehuddin, A., et al. (2006). Early outcome of reamed interlocking nail for non union of tibia. Med J. Malaysia. 61 (3): 339-342

[10] Said, G., El Sharkawi, M., Said, H., et al. (2011) Fibula-pro-tibia in plating tibial non-unions. Int Orthop. 35 (11): 1713-1718

[11] Wu, C., Shih, C., Chen, W., et al. (1999). High success rate with exchanging to treat a tibial shaft aseptic non union. J. Orthop Trauma. 13: 33-38.

[12] Ghaffarpasand, F., Shahrezaei, M., Dehghankhalili M. (2016). Effects of platelet rich plasma on healing rate of long bone non-union fractures: A randomized double-blind placebo controlled clinical trial. Bull Emerg Trauma. 4 (3): 134-140. 